\title{
Separation properties of the MIL-125(Ti) Metal-Organic Framework in high-performance liquid chromatography revealing cis/trans selectivity
}

\author{
Stijn Van der Perre ${ }^{a}$, Anuschka Liekens ${ }^{a}$, Bart Bueken ${ }^{b}$, Dirk E. De Vos ${ }^{b}$, Gino V. Baron ${ }^{a}$, \\ Joeri F.M. Denayer ${ }^{\mathrm{a}, *}$ \\ a Department of Chemical Engineering, Vrije Universiteit Brussel, Pleinlaan 2, Brussel B1050, Belgium \\ ${ }^{\mathrm{b}}$ Centre of Surface Chemistry and Catalysis, Katholieke Universiteit Leuven, Celestijnenlaan 200F - box 2461, Leuven 3001, Belgium
}

\section{A R T I C L E I N F O}

\section{Article history:}

Received 26 July 2016

Received in revised form

15 September 2016

Accepted 23 September 2016

Available online 24 September 2016

\section{Keywords:}

Metal-Organic Frameworks

MIL-125(Ti)

Modulated synthesis

cis/trans selectivity

van Deemter

\begin{abstract}
A B S T R A C T
Monodisperse MIL-125(Ti) Metal-Organic Framework crystals were synthesized and studied as stationary phase in high performance liquid chromatography (HPLC). Different pure compounds and model mixtures (including stereoisomer mixtures) were injected from which chromatographic parameters, including selectivities and resolution factors, were determined to evaluate the adsorption properties and separation performance of MIL-125(Ti) in liquid phase. The MIL-125(Ti) framework displayed a trans selectivity for cis/trans difunctionalized cyclohexane molecules with high selectivity and resolution for 1,3-dimethylcyclohexane and 4-ethylcyclohexanol. The slurry-packed column was further characterized via van Deemter analysis. Fitting of the van Deemter equation through the experimental points allowed to define the contributions of the different processes to plate height with a significant proportion of the A-term, reflecting the importance of a good crystal packing. Although high in comparison to commercial HPLC stationary phases, a very good plate height of around $50 \mu \mathrm{m}$ was found.
\end{abstract}

(c) 2016 Elsevier B.V. All rights reserved.

\section{Introduction}

Since the introduction of Metal-Organic Frameworks (MOFs) in the area of adsorption, the study of these porous crystalline structures in liquid and gas phase chromatography has already demonstrated interesting separation properties [1,2]. In comparison with more conventional stationary phases like (functionalized) silica or alumina in high-performance liquid chromatography (HPLC), MOFs can lead to chromatographic separations based on molecular size and shape due to a uniform pore size distribution and ordered pore structure. Hereby, the versatility of organic linkers and metal centers used in MOFs introduce other interactions with the analytes than commercial stationary phases in HPLC, which opens possibilities to separate different types of mixtures.

A series of traditional MOFs, such as HKUST-1 [3,4], MOF-5 [3], MIL-47(V) [4], MIL-53(Al/Fe) [4-8], MIL-100(Fe) [8,9], MIL-101(Cr) $[8,10,11]$, MIL-125(Ti) $\left(-\mathrm{NH}_{2}\right)[12,13]$ and UiO-66(Zr) [14] have been tested as stationary phase for powder packed columns in HPLC. In most cases, the separation of different aromatic positional isomers (xylene, dichlorobenzene, chlorotoluene, chloroaniline, toluidine,

\footnotetext{
* Corresponding author.

E-mail address: Joeri.Denayer@vub.ac.be (J.F.M. Denayer).
}

cresol, nitrophenol, nitroaniline and aminophenol) and polycyclic aromatic hydrocarbons (PAHs) was studied to underline their separation performance.

Drawbacks of these columns are high pressure drops, low column performance and pronounced peak broadening due to the polydispersity of most MOF particles and their non-spherical, irregular shape. To increase column efficiency and avoid undesirable peak broadening, silica-MOF composites were proposed as stationary phase, combining the packing properties of spherical silica particles and the separation ability of MOF crystals. In that context, HKUST-1 [15,16], ZIF-8 [17,18], UiO-66(Zr) [19,20] and a chiral MOF [21] were evaluated as hybrid silica-MOF packing material for liquid chromatography. Some of these MOFs were even implemented as core-shell particles, where a porous MOF layer is grown around a solid silica core [16-18,20], to further improve chromatographic performance. This has led to faster and high-resolution separations with high efficiencies, reaching around 18,000, 23,000 and 19,000 plates/m for aromatic compounds on HKUST-1@SOS [16], ZIF-8@SiO 2 [17] and ZIF-8@SOS (SOS particles are nonporous silica microspheres with a spheres-on-sphere morphology) [18], respectively.

Another obvious way to circumvent the disadvantages of columns packed with irregular MOF particles is the synthesis of monodisperse MOF particles of spherical shape. Nevertheless, this 
(a)

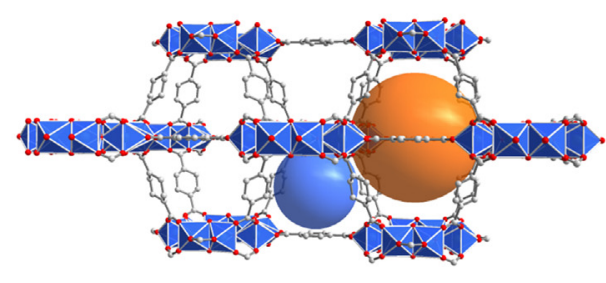

(b)

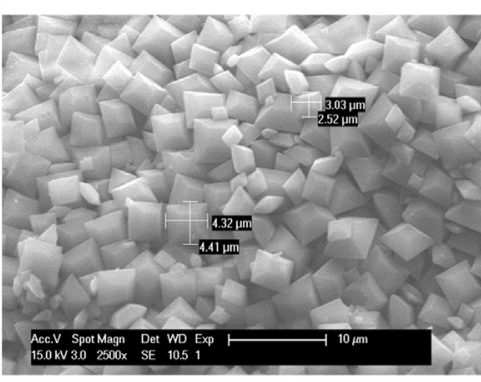

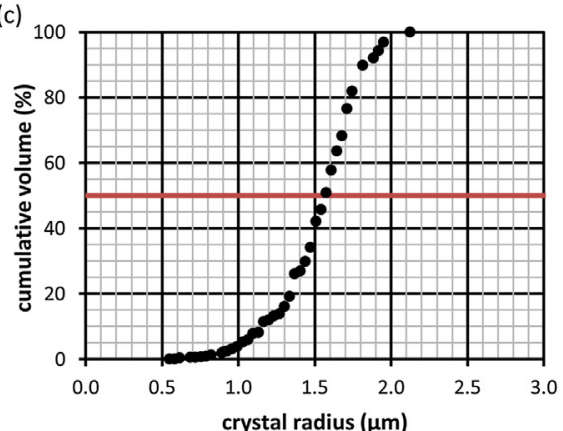

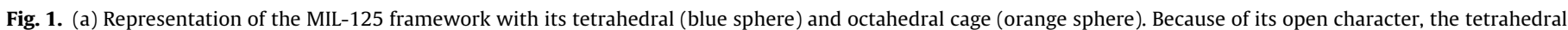

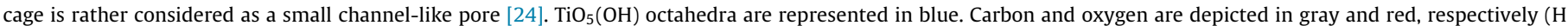

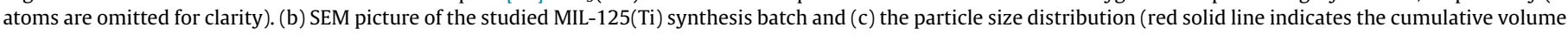
at 50\%). (For interpretation of the references to color in this figure legend, the reader is referred to the web version of this article.)

is not as straightforward as might seem, since the final crystal morphology is determined by the intrinsic crystal structure, often resulting in needle- or platelet-like morphologies. In this perspective, MIL-125(Ti) is an interesting material. It is a Ti-based MOF, built up from cyclic octamers (SBU-8) of edge- and corner-sharing $\mathrm{TiO}_{5}(\mathrm{OH})$ octahedra connected by 1,4-benzenedicarboxylate (BDC) linkers. MIL-125(Ti) possesses distorted octahedral (12.5 $\AA$ ) and tetrahedral (6.1 $\AA$ ) cages connected with triangular windows of 5-7 Å in size [22] (Fig. 1a). Standard synthesis of MIL-125(Ti) delivers crystals which are thin and disk-like in shape, with a size between 4 and $5 \mu \mathrm{m}$ [22]. Hu et al. [23] developed a synthesis method for MIL-125(Ti)- $\mathrm{NH}_{2}$ with controllable morphology via a solvothermal reaction just by modulating reactant concentration. Different morphologies from circular plate to octahedron were obtained.

The MIL-125(Ti) and its amine variant have already been tested as separation medium for different isomer mixtures with a pronounced para-selective character for the separation of xylene isomers in liquid phase [12]. Based on simulation data and an experimental analysis, the authors proposed that this selectivity follows from packing effects in the large octahedral cages and molecular sieving effects in the small tetrahedral cages. Moreira et al. described the effect of ethylbenzene on the separation of xylene isomers in quaternary breakthrough experiments with MIL-125(Ti) [13]. This material was also investigated for the separation of hexane isomers via molecular simulations, where it was found that the MIL-125(Ti) structure is selective towards the linear molecule [24]. Contrary to UiO-66, possessing closed tetrahedral cages, the tetrahedral cages of MIL-125(Ti) are open (cross-shaped pore space) and act as a sort of small, channel-like pores (Fig. 1a). This means $n$-hexane can be adsorbed while conserving its linear conformation in the open tetrahedral cages [24], whereas in the UiO-66 the dibranched molecules are better confined in the closed tetrahedral cages [24-26]. MIL-125(Ti) and MIL-125(Ti)- $\mathrm{NH}_{2}$ have also been proposed by Kim et al. [27] for the separation of isoprene from 2methylbutane, a mixture which is typically produced as a mixture in a steam cracker. Both materials exhibit a two-step isotherm for both adsorbates due to the presence of two types of cages, with higher adsorption capacities for isoprene. Based on water vapor isotherms on these materials, the amino variant of MIL-125(Ti) is far more hydrophilic and appears to be more stable to water than the unfunctionalized framework [27]. The MIL-125(Ti)- $\mathrm{NH}_{2}$ material was even successfully investigated as humidity sensor by Zhang et al. $[28,29]$.

In this work, a method was developed to synthesize monodisperse, nearly spherical MIL-125(Ti) particles. This material was used as stationary phase in liquid chromatography. Its separation performance was studied using different compounds including difficult-to-separate stereoisomer mixtures. The slurry packed MIL-125(Ti) column showed relatively high selectivity and resolution for the separation of structural aromatic isomers, cis/trans cyclohexane isomers and specific model mixtures. Furthermore, mass transfer properties of the stationary phase, in the form of crystallites, were characterized by a van Deemter curve.

\section{Materials and methods}

\subsection{Synthesis of monodisperse MIL-125(Ti) crystals}

MIL-125(Ti) crystals were prepared by applying the concept of synthesis modulation using a monocarboxylic acid additive [30], in this case acetic acid, to a previously reported synthesis protocol for MIL-125 [22,31]. In a typical synthesis, $2.4 \mathrm{~g}(1.13 \mathrm{mmol})$ of pre-formed octameric Ti-pivalate clusters was dissolved in a mixture of $50 \mathrm{ml} \mathrm{N}, \mathrm{N}$-dimethylformamide(DMF) and $20 \mathrm{ml}$ of methanol $(\mathrm{MeOH})$ in a $250 \mathrm{ml}$ pyrex Schott bottle. To this, a solution of $3 \mathrm{~g}(18 \mathrm{mmol})$ of terephthalic acid dissolved in $50 \mathrm{ml}$ of DMF was added. Glacial acetic acid $(3.1 \mathrm{ml} ; 54 \mathrm{mmol} ; 3$ molar equivalents with respect to terephthalic acid) was added to this mixture, after which the synthesis mixture was allowed to react at $393 \mathrm{~K}$ for $48 \mathrm{~h}$. The resulting solid was recovered by centrifugation. Unreacted terephthalic acid was removed from the pores by soaking the solid twice overnight in DMF at $393 \mathrm{~K}$, followed by three solvent exchange steps with ethanol overnight at $333 \mathrm{~K}$. Finally, ethanol was removed from the pores by a thermal treatment overnight at $423 \mathrm{~K}$ in air.

Relatively monodisperse particles with a specific morphology were obtained, shaped as truncated octahedral crystals (truncated at top and bottom), having an average size around $3 \mu \mathrm{m}$, as shown in Fig. 1b. Particle size distribution was determined via Scanning Electron Microscopy image analysis (Fig. 1c), where the average crystal size is calculated on basis of the cumulative volume at $50 \%$. These crystals were approximated as ellipsoids. From spheres with identical volume as the ellipsoids, the corresponding crystal radii were calculated. Ar porosimetry (Quantachrom Autosorb-IQ instrument) revealed a total pore volume of $0.68 \mathrm{ml} / \mathrm{g}$, while X-ray diffraction (XRD) analysis was carried out to verify the crystalline structure (Fig. S3).

\subsection{Preparation of the MIL-125(Ti) packed column}

A 2 wt-\% slurry of MIL-125(Ti) as the stationary phase in $n$ hexane was prepared in an ultrasonically agitated reservoir. Using a slurry packer, a stainless steel column (length $100 \mathrm{~mm}$, inner diameter $2.1 \mathrm{~mm}$ ) was packed and allowed to settle at $400 \mathrm{bar}$ for a few hours using acetonitrile $(\mathrm{ACN})$ as the displacement liq- 
uid. Afterwards, the HPLC column was flushed with ACN at a flow of $0.2 \mathrm{ml} / \mathrm{min}$ for $2 \mathrm{~h}$ on the HPLC device before chromatographic experiments. HPLC experiments were performed with an Agilent Technologies 1100 series apparatus, equipped with UV-vis and RI (refractive index) detector.

\subsection{Performance evaluation of the crystallite packed HPLC column}

Retention times $t_{r}$, peak widths $\left(w_{b}\right.$ and $\left.w_{h}\right)$ and peak symmetries $\left(A_{s}\right)$ were obtained via the chemstation software. The void time, $t_{0}$, to calculate the retention factor $k$ ' was determined by injecting a small plug of ACN in RP mode (ACN) or $n$-heptane in $\mathrm{NP}$ ( $n$-heptane) mode and recording the perturbation signal.

\subsection{Analytes and mobile phase solvents}

Chromatographic experiments were performed with different chemical families, including aromatic positional isomers, polycyclic aromatic hydrocarbons (PAHs), aryl halides, alkyl and hetero functionalized aromatics, and cis/trans functionalized cyclohexane compounds. Ultrapure water and HLPC gradient solvents (ACN, $\mathrm{MeOH}$ and $n$-heptane) were used as mobile phase. A more detailed overview with purity and provider of each component is found in Supporting information.

The dimensions of different analytes (including van der Waals radii) were determined by the ChemDraw software (ChemBio3D Ultra). Firstly, an optimization of the structure was performed in the software by minimizing its energy, resulting in its most ideal conformation. An overview of the molecular dimensions is given in Table S1 in Supporting information.

\section{Results and discussion}

A variety of analytes with different structural and chemical properties, such as aromatic positional isomers, polycyclic aromatic hydrocarbons (PAHs), compounds with polar functionalities, aryl halides, chloro-substituted compounds, and stereoisomer mixtures (cis/trans isomers) have been injected to explore the separation performance of the MIL-125(Ti) structure in liquid phase. All of these compounds and mixtures were dissolved in ACN. It is a polar solvent which is commonly used in combination with water as an organic modifier in reversed phase (RP) chromatography (in the case of hydrophobic stationary phases) [33]. But water had a detrimental effect on the pore structure of MIL-125(Ti) (Fig. 2); a decrease of $60 \%$ in pore volume was noticed after using an aqueous mobile phase, which is ascribed to a drastic change in the pore structure of MIL-125(Ti) (by collapsing of the cages or to partial blocking of certain pores). This hydro-instability was also mentioned by Kim et al. [27]. Therefore, pure ACN was used as mobile phase. Separations in other mobile phase compositions are described in Supporting information.

\subsection{Separation of aromatic positional isomers}

Since a para-selective behavior was assigned to the isostructural MIL-125- $\mathrm{NH}_{2}$ and CAU-1(Al)- $\mathrm{NH}_{2}$ materials, the chromatographic separation of xylene and methylacetophenone isomers was studied on the MIL-125(Ti) packed column. For the separation of mixtures of xylene, ethyltoluene and cymene isomers on MIL-125(Ti)- $\mathrm{NH}_{2}$, para isomers were clearly preferred in pulse chromatographic experiments performed by Vermoortele et al. [12]. In the present study, using the non-functionalized form of the material, explicit para selectivity is found for xylenes and methylacetophenone isomers, as illustrated by the longer retention of these compounds in the chromatograms in Fig. 3, resulting in separation factors (a)

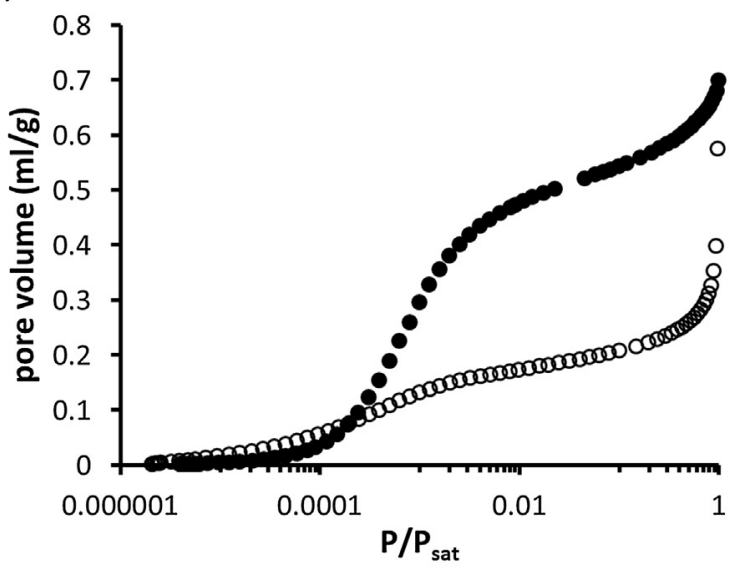

(b)

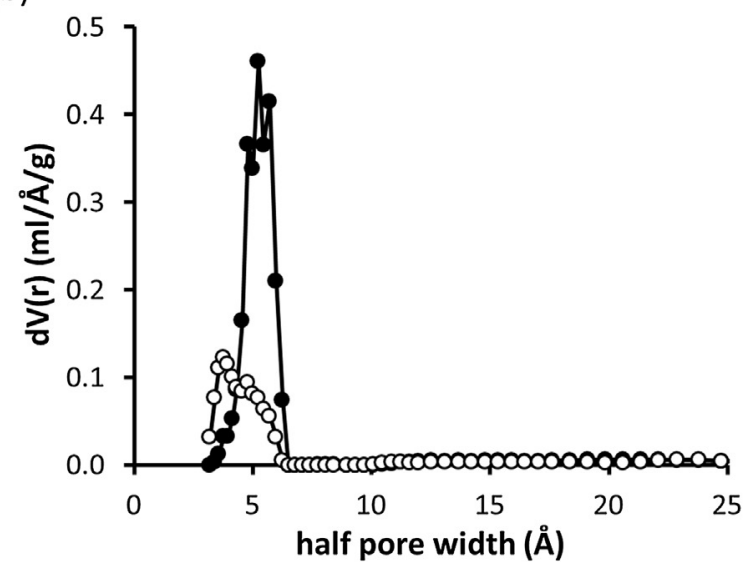

Fig. 2. (a) Ar uptake at $87 \mathrm{~K}$ and (b) DFT pore size distribution of the original MIL125(Ti) (closed symbols) and after contact with water (open symbols).

between 1.1 and 2.1 (see Table 1 ). In the case of the xylenes, the MIL-125 framework hardly differentiates between $m$ - and $o$-xylene with a slight preference for $o$-xylene, which was also seen by Moreira et al. [13]. For larger aromatic structural isomers, like methylacetophenone, the difference in retention between the isomers is larger. The peaks of these compounds are fairly symmetric and relatively narrow for a microporous material. High selectivity and resolution factor is attained for the para isomer relative to the meta- and ortho-analogue (Table 1 ). The elution order (ortho<meta<para) is perfectly in accordance with the dimensions of the molecules, in particular the increasing length $(9.5 \AA<10.1 \AA<10.6 \AA)$ and decreasing width $(7.8 \AA>7.3 \AA>6.6 \AA)$ (Table S1). As shown earlier in simulation studies for the separation of linear and branched alkanes on the MIL-125(Ti) structure [24], the open tetrahedral cages favor the adsorption of more linear structures, conforming the better interaction with ellipsoidally shaped isomers.

\subsection{Separation of polycyclic aromatic hydrocarbons (PAHs)}

The study of the separation of different PAHs, such as pyrene, indene, naphthalene, phenanthrene and anthracene, reveals a strong dependency of retention time on both the molecular size and shape (Fig. 4). The retention time increases in the order: pyrene $<$ indene $=$ naphthalene $<$ phenanthrene $<$ anthracene. The least bulky molecule (smallest kinetic diameter) with the largest number of rings thus is the most strongly retained. In spite of a difference of one carbon atom, indene and naphthalene, with 
Table 1

Retention k', asymmetry $A_{s}$, selectivity $\alpha$ and resolution factors $\mathrm{R}_{\mathrm{S}}$ for different chromatographic separations.

\begin{tabular}{|c|c|c|c|c|}
\hline & $\mathrm{k}^{\prime}(-)$ & $A_{S}(-)$ & $\alpha(-)$ & $\mathrm{R}_{\mathrm{S}}(-)$ \\
\hline \multicolumn{5}{|l|}{ Xylene isomers } \\
\hline $\mathrm{p} / \mathrm{o}$ & $1.24 / 0.71$ & $2.87 / 2.68$ & 2.13 & 2.38 \\
\hline $\mathrm{o} / \mathrm{m}$ & 0.63 & 2.76 & 1.14 & - \\
\hline \multicolumn{5}{|l|}{ Methylacetophenone isomers (overlay) } \\
\hline $\mathrm{p} / \mathrm{m}$ & $2.33 / 1.14$ & $1.89 / 2.29$ & 2.05 & 4.06 \\
\hline $\mathrm{m} / \mathrm{o}$ & 0.87 & 1.71 & 1.28 & 0.94 \\
\hline \multicolumn{5}{|l|}{ PAHs (overlay) } \\
\hline anthracene/phenanthrene & $5.94 / 5.06$ & $2.72 / 2.05$ & 1.25 & 1.45 \\
\hline phenanthrene/naphthalene & 0.94 & 2.46 & 2.47 & 4.79 \\
\hline naphthalene/indene & 0.92 & 2.41 & 1.01 & 0.08 \\
\hline indene/pyrene & 0.14 & 8.49 & 6.66 & 5.47 \\
\hline \multicolumn{5}{|l|}{ Aryl halides (overlay) } \\
\hline iodo-/bromobenzene & $1.97 / 1.49$ & $2.44 / 2.60$ & 1.32 & 2.11 \\
\hline bromo-/chlorobenzene & 1.22 & 2.63 & 1.22 & 1.38 \\
\hline chloro-/fluorobenzene & 0.87 & 2.81 & 1.41 & 1.88 \\
\hline \multicolumn{5}{|l|}{ Molecules with polar functionalities } \\
\hline phenol/benzyl alcohol (overlay) & $8.95 / 2.29$ & $5.12 / 4.68$ & 3.92 & 4.31 \\
\hline benzyl alcohol/benzaldehyde (overlay) & 1.70 & 3.54 & 1.34 & 1.31 \\
\hline benzaldehyde/benzene (overlay) & 1.12 & 2.92 & 1.52 & 1.96 \\
\hline cyclohexanol/cyclohexanone & $1.64 / 0.66$ & $4.14 / 2.05$ & 2.46 & 2.87 \\
\hline \multicolumn{5}{|l|}{ Small molecules (overlay) } \\
\hline styrene/ethylbenzene & $1.62 / 1.19$ & $2.19 / 2.32$ & 1.21 & 1.01 \\
\hline cycloheptane/cyclohexane & $2.40 / 1.19$ & $2.51 / 2.27$ & 2.01 & 3.66 \\
\hline cyclohexane/cyclohexene & $1.19 / 0.65$ & $2.27 / 1.89$ & 1.79 & 2.39 \\
\hline methylcyclohexane/toluene & $1.22 / 0.74$ & $1.96 / 2.00$ & 1.66 & 2.33 \\
\hline \multicolumn{5}{|l|}{ Large molecules (overlay) } \\
\hline 3-bromobiphenyl/2-bromo-1-phenylpropane & $3.41 / 1.99$ & $1.94 / 2.00$ & 2.15 & 5.77 \\
\hline 2-bromo-1-/1-bromo-3-phenylpropane & 1.74 & 2.08 & 1.15 & 0.94 \\
\hline dibenzyl ether/3-bromobiphenyl & $3.51 / 3.41$ & $1.68 / 1.94$ & 1.06 & 0.58 \\
\hline 3-bromobiphenyl/dibenzothiophene & 2.18 & 1.68 & 1.94 & 3.00 \\
\hline \multicolumn{5}{|l|}{ Stereoisomer mixtures(trans/cis) } \\
\hline 1,3-dimethylcyclohexane & $0.92 / 0.47$ & $1.86 / 1.91$ & 1.96 & 1.97 \\
\hline 1,4-dimethylcyclohexane & $0.94 / 0.61$ & $-/ 2.04$ & 1.52 & 1.47 \\
\hline 4-ethylcyclohexanol & $1.82 / 0.81$ & $3.21 / 3.74$ & 2.25 & 3.16 \\
\hline dimethyl 1,4-cyclohexanedicarboxylate & $0.47 / 0.20$ & $-/ 1.86$ & 2.30 & 1.23 \\
\hline
\end{tabular}

their similar size, elute simultaneously. This demonstrates that for apolar molecules without specific functionalities, molecular weight or number of carbon atoms is not the main factor in the retention behavior. Given the very similar shape and size of both molecules (Table S1), it can be concluded that these properties are critical in the adsorptive interactions in the confined environment of the MIL-125(Ti) pores. The extra ring in phenanthrene and anthracene compared to naphthalene provides more occasions to interact with the pore wall, resulting in a convincing longer retention. The bulky pyrene molecule starts eluting very rapidly, with a retention time very close to the dead time, but has a very tailed peak. This indicates that this molecule is at the border of being excluded from adsorption in the MIL-125(Ti) pores, just like 1,3,5-triisopropylbenzene and 1,3,5-tri-tertbutylbenzene (Table S1). The open tetrahedral cages accommodate very well long and slim molecules while the pore openings impose steric restrictions on the adsorption of molecules with multiple rings in a non-linear configuration.

\subsection{Separation of molecules with polar functionalities}

The chromatographic separation of model mixtures, where the effect of functional groups was studied instead of molecular shape, revealed specific interactions of the framework with functional groups (Fig. 5). Significant differences in retention for very similar molecules are observed, whereby the presence of a functional group has a major impact on retention (Fig. 5a). Retention of benzaldehyde, benzyl alcohol and phenol increased 30, 55 and 370\%, respectively, as compared to their non-functionalized analogue, benzene. This points at the occurrence of specific interactions between the adsorbates and the stationary phase. It has been shown previously that the corner-sharing hydroxyl groups $\left(\mu_{2}-\mathrm{OH}\right)$ on the octanuclear entities $\left(\mathrm{Ti}_{8} \mathrm{O}_{8}(\mathrm{OH})_{4}\right)$ play an important role in the adsorption of certain molecules via the formation of hydrogen bonds. In a recent study, Vaesen et al. pointed at hydrogen bonding between the $\mu_{2}-\mathrm{OH}$ groups (H-donor) and the sulfur atom of $\mathrm{H}_{2} \mathrm{~S}$ (H-acceptor) via in situ IR spectroscopy during the adsorption of $\mathrm{H}_{2} \mathrm{~S}$ on MIL-125(Ti) [34]. Such specific interactions between guest molecules and $\mu_{2}-\mathrm{OH}$ hydroxo groups (framework hydroxyl groups) were also found in another member of the MIL family, where hydrogen bonding between water molecules and those hydroxyl groups forms the basis of the breathing behavior of the MIL-53 structure, i.e. the opening and closing of the pore structure triggered by host-guest interactions [35-37]. A positive effect of the hydroxyl group present on the adsorbate in terms of retention is noticed in comparison with other functional groups, such as an aldehyde $(-\mathrm{CHO})$ or carbonyl $(-\mathrm{C}=\mathrm{O})$ functionality (Fig. 5a and b, respectively). Similar behavior was found in liquid chromatographic experiments on silica and alumina, where the hydroxyl group has a stronger interaction (higher group adsorption energy) with the adsorbent than an aldehyde or carbonyl group. Depending on whether the functional group is attached to an aliphatic or aromatic residue, a different adsorption energy has been allocated to the molecules $[38,39]$. The stronger interaction of these functional groups with the framework mainly results from the ability of hydrogen bonding with the framework [38]. Besides hydrogen bonding, also solubility of the solute in the mobile phase and the strength of the mobile phase interactions with the adsorbent determine the retention behavior. The strength of retention is increased by introduction of a second functional group, which is the case for methylparaben (also called methyl 4-hydroxybenzoate), displaying a hydroxyl $(-\mathrm{OH})$ and ester $(-\mathrm{COOR})$ entity. It showed longer retention than phenol in this mobile phase $\left(k^{\prime}=17.3\right.$ versus 8.9 , see Fig. S6). 

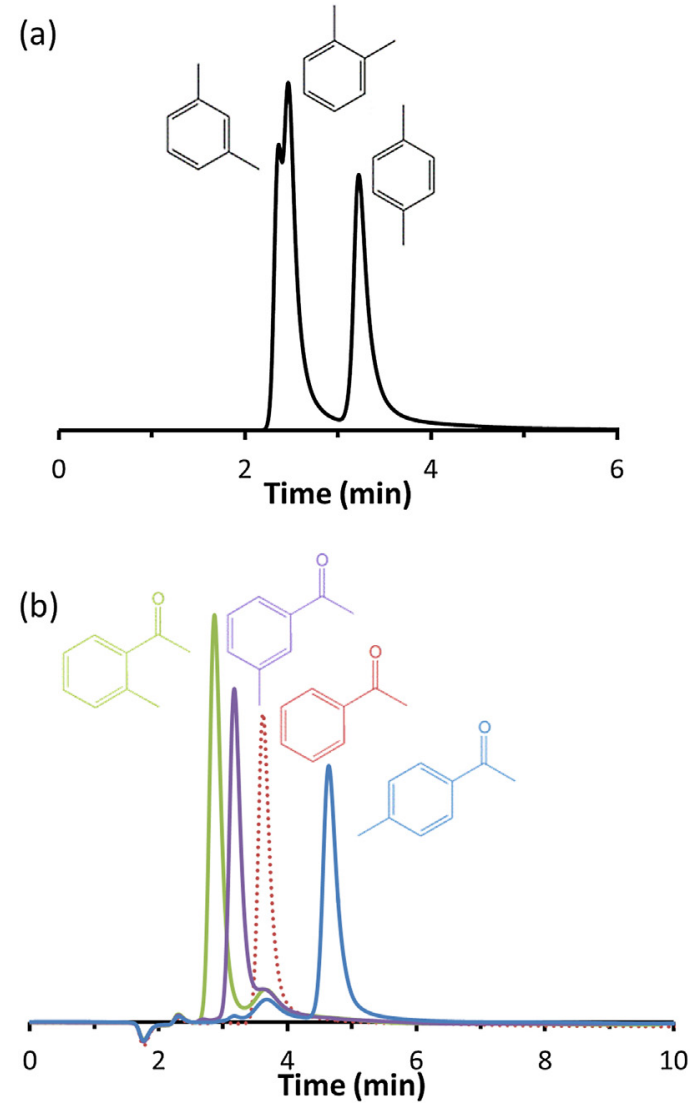

Fig. 3. Chromatographic profiles of (a) a mixture of $m-, \quad o$ - and $p$-xylene (33.3/33.3/33.3 composition) and (b) overlay of $o$ - (green), $m$ - (purple) and $p$ methylacetophenone (blue) chromatograms on MIL-125(Ti) using a mobile phase of ACN at $303 \mathrm{~K}$ and a flow rate of $0.20 \mathrm{ml} / \mathrm{min}$. The small peaks in (b), around $3.6 \mathrm{~min}$, point at the presence of an impurity of acetophenone, which is confirmed by an extra injection (red dotted line). (For interpretation of the references to color in this figure legend, the reader is referred to the web version of this article.)

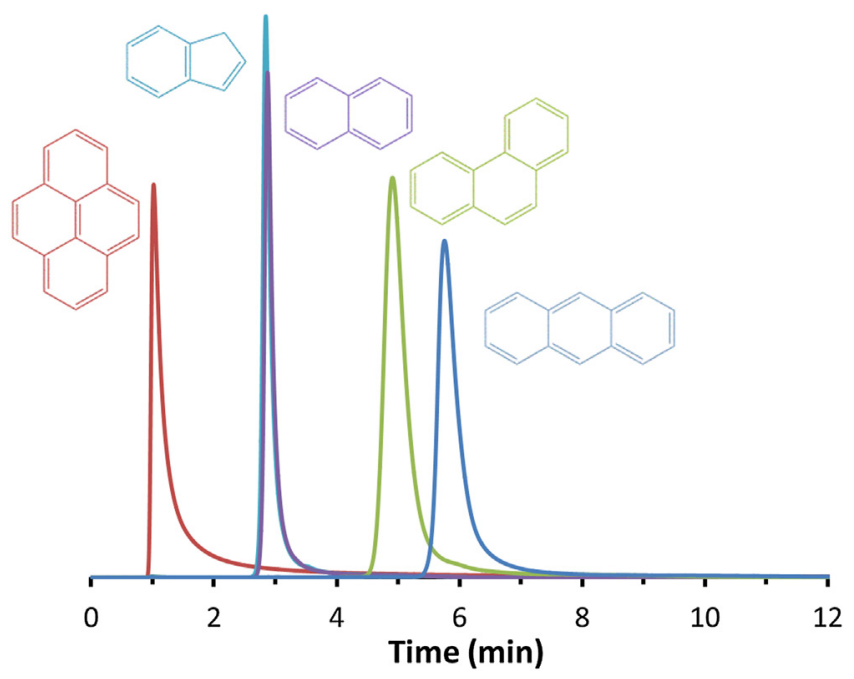

Fig. 4. Chromatographic profiles (overlay) of pyrene (red), indene (light blue), naphthalene (purple), phenanthrene (green) and anthracene (blue) on MIL-125(Ti) using a mobile phase of ACN at $303 \mathrm{~K}$ and $0.20 \mathrm{ml} / \mathrm{min}$. (For interpretation of the references to color in this figure legend, the reader is referred to the web version of this article.) (a)

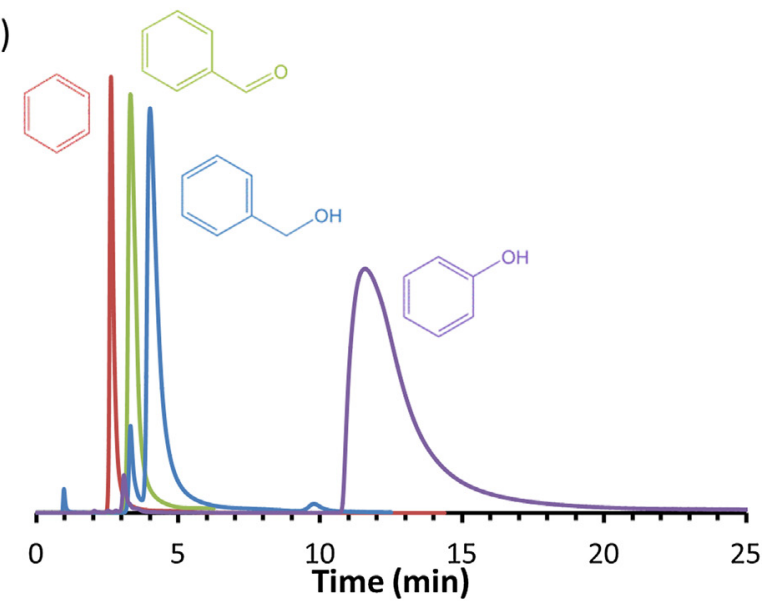

(b)

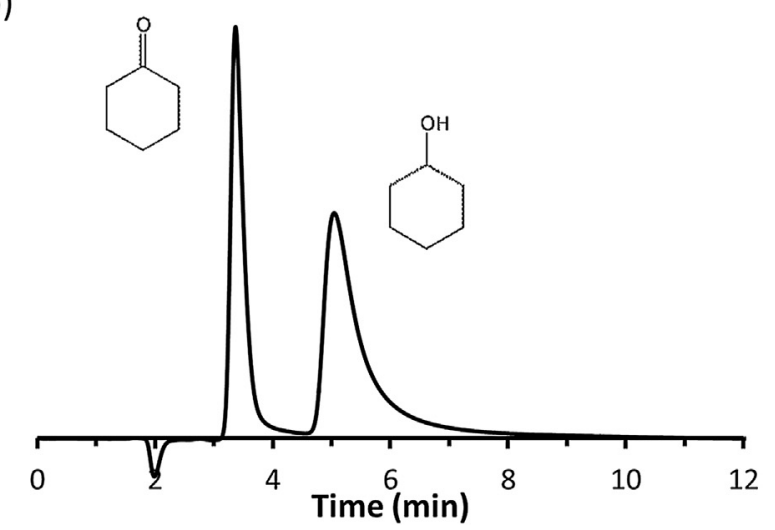

Fig. 5. Chromatographic profiles of (a) benzene (red), benzaldehyde (green), benzyl alcohol (blue) and phenol (purple) and (b) a mixture of cyclohexanone and cyclohexanol (50/50 composition) on MIL-125(Ti) using a mobile phase of ACN at $303 \mathrm{~K}$ and $0.20 \mathrm{ml} / \mathrm{min}$. (For interpretation of the references to color in this figure legend, the reader is referred to the web version of this article.)

\subsection{Separation of aryl halides}

Furthermore, different aryl halides and chloro-substituted benzene compounds were injected to study the effect of halogenic groups on adsorption (Fig. 6). Adsorbates of the monohalogen aromatic series elute sequentially in order of molecular weight $(-\mathrm{F}<-\mathrm{Cl}<-\mathrm{Br}<-\mathrm{I}$; see Fig. 6a), which means that retention increases with polarizability of these compounds. This is not unexpected, since the dispersion forces become larger with increasing polarizability [40]. Also, the substitution of a methyl-group or $\mathrm{H}$-atom by a $\mathrm{Cl}$-atom enhanced the interaction with the framework via halogen bonding with framework oxygens/hydroxyl groups/ $\pi$-systems [41] or via weak $\mathrm{CH} \cdots \mathrm{Cl}$ hydrogen bonds [42] (Fig. 6b-d).

\subsection{Separation of stereoisomers}

Subsequently, the separation of cis/trans alkyl functionalized cyclohexane mixtures was studied. A clear preference for adsorbing the trans isomer was demonstrated for 1,3- and 1,4dimethylcyclohexane, with (almost) baseline separation of the isomers (Fig. 7a,b). The same trend was observed in case of heterosubstituted cyclohexane molecules, such as 4-ethylcyclohexanol and dimethyl 1,4-cyclohexanedicarboxylate, with an explicit advantage for the trans configuration (Fig. 7c,d). High selectivity and resolution factor were obtained for 1,3-dimethylcyclohexane 
(a)

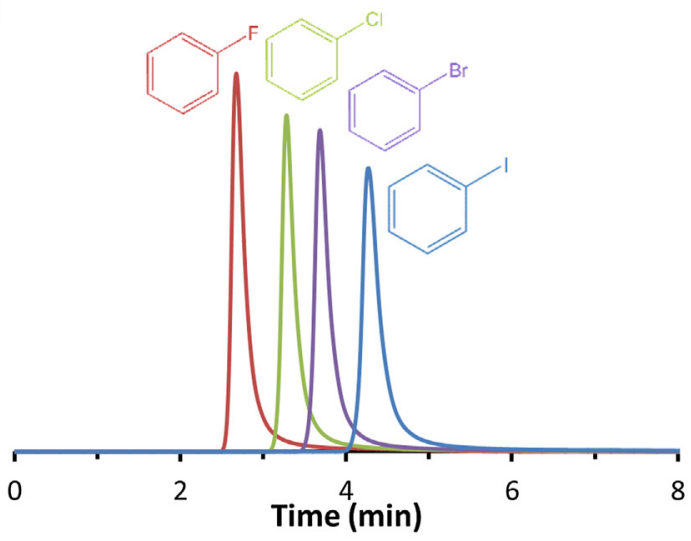

(c)

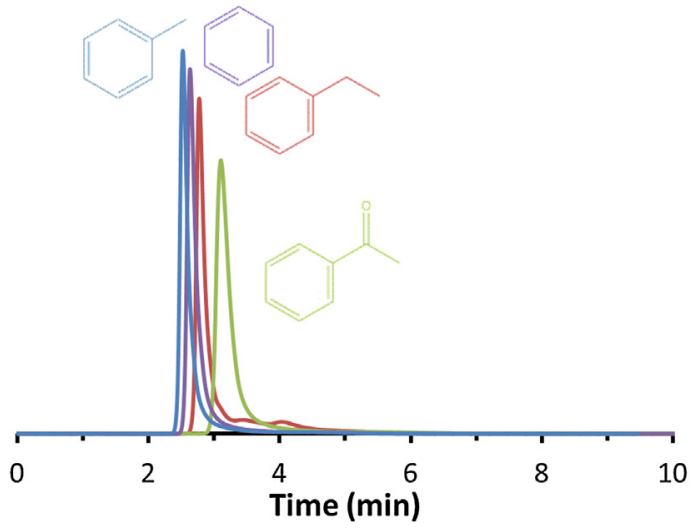

(b)

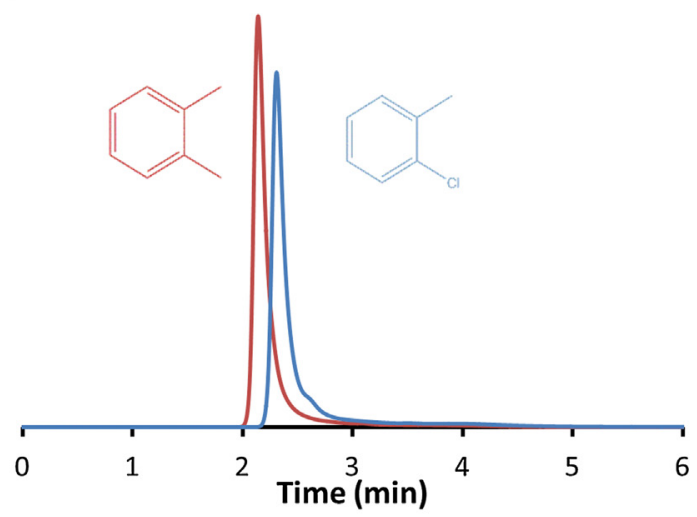

(d)

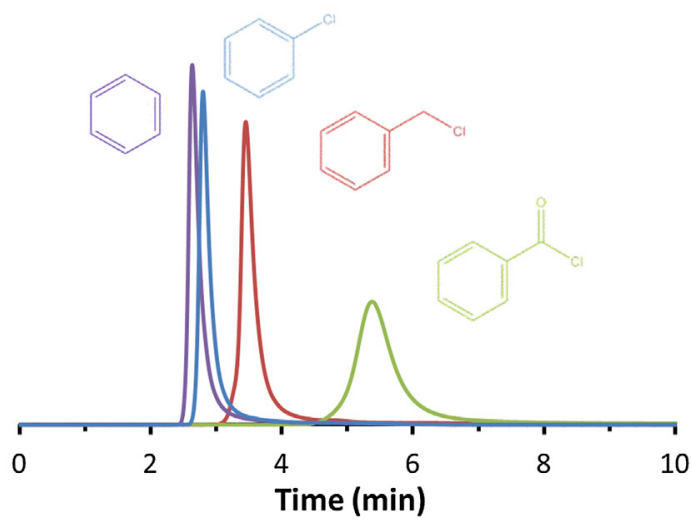

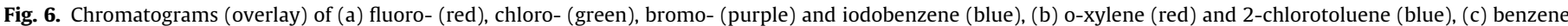

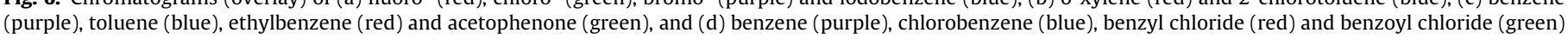

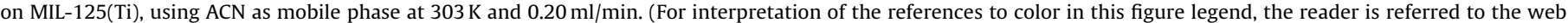
version of this article.)

and 4-ethylcyclohexanol isomer mixtures (Table 1). Due to their nearly identical physicochemical properties, the chromatographic separation of these stereoisomers is extremely difficult using classical stationary phases (Fig. S10). The separation of this type of molecules is of great importance for the synthesis and development of certain pharmaceuticals, where cis and trans isomers can possess a totally different biological activity [43-45].

The tendency of favoring the trans isomer is explained by the preference for molecules with a more elongated and slimmer shape (assuming the most stable conformation, i.e. with substituents in equatorial position), which fit and interact better in the open tetrahedral cage, acting as a channel between the large octahedral cages. This is less straightforward for 1,3dimethylcyclohexane, where the cis isomer has a larger maximum length, but has also a significantly larger width than the trans isomer (Table S1). This results in an easy fit of the trans isomer into the cross-shaped pore space while the cis isomer is probably sterically hindered. The ortho variant of dimethyl substituted cyclohexane (1,2-dimethylcyclohexane) could not be separated and a broad peak was obtained for this cis/trans mixture (Fig. S9a) as a consequence of the marginal differences in molecular dimensions between the 1,2-dimethylcyclohexane isomers, in contrast to 1,3- and 1,4-dimethylcyclohexane. Cis/trans mixtures of hetero-functionalized cycloalkanes, such as 1,4-cyclohexanediol, 1,4-cyclohexanedimethanol, 2-chlorocyclohexanol, 2,5-dimethyltetrahydrofurane, 3methylcyclohexanol and 4-methyl-1-cyclohexanemethanol, were injected as well, but could not be separated efficiently. Only for 4- methyl-1-cyclohexanemethanol and 1,4-cyclohexanedimethanol, where the difference in molecular dimensions between the stereoisomers is more pronounced (Table S1), a small differentiation between cis and trans isomers could be perceived as a shoulder in the peak (Fig. S9b and c). In all other cases, a single (broader) peak was obtained. Also, separation of a stereoisomer mixture of 1,3-pentadiene (annotated as $\mathrm{E} / \mathrm{Z}$ isomers instead of cis/trans) was evaluated on the MIL-125(Ti) column. The molecular dimensions of these diene isomers are probably too small compared to the tetrahedral pore space to obtain a shape selective separation (Fig. S9d).

In previous work, it was reported that UiO-66, also a structure with tetrahedral and octahedral cages, could perform cis/trans separations of similar molecules (difunctionalized cyclohexanes) in gas and liquid chromatography $[14,25]$. In this material, having tetrahedral cages with a more closed character, a reversed selectivity was found, showing preference for the most bulky isomer (substituents in the axial and equatorial position which corresponds to the cis isomer in the 1,2 and 1,4 position, and the trans isomer in the 1,3 position). In this MOF, the more compact molecules are fitting better in the closed tetrahedral cages, resulting in a better interaction with the UiO-66 framework.

\section{6. van Deemter analysis}

Because good separation performance together with acceptable peak shape and broadening was obtained for this MIL-125(Ti) crystal packed column, the column was characterized via a van Deemter 
(a)

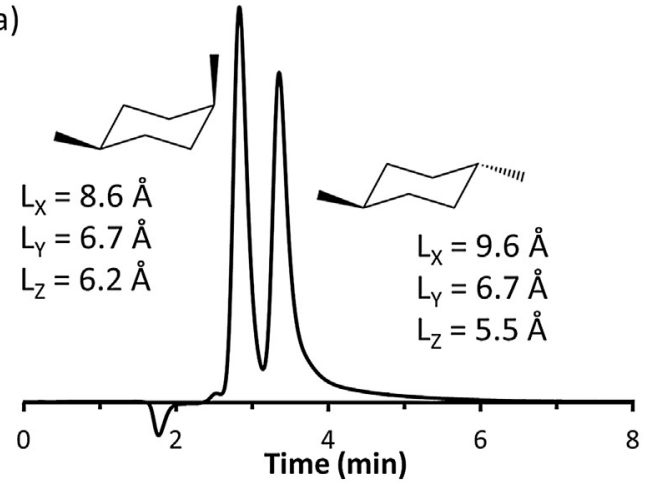

(c)

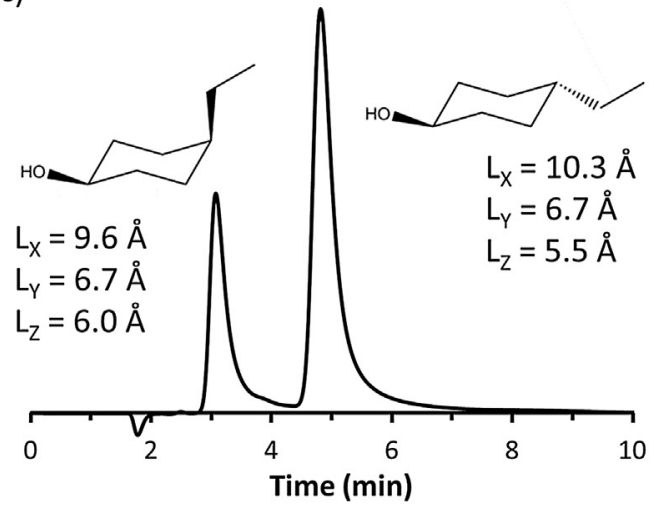

(b)

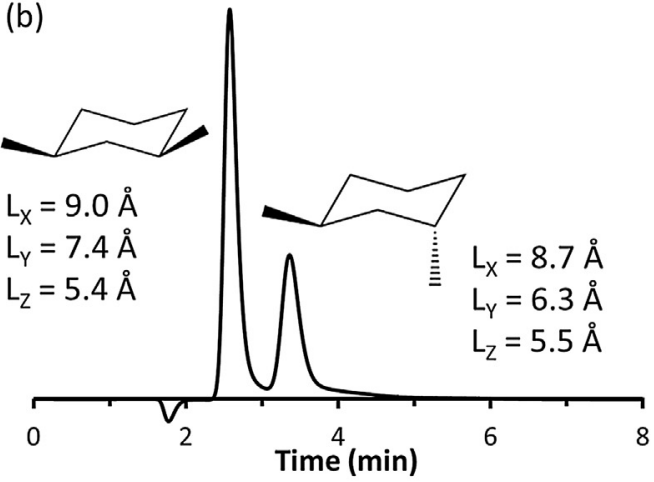

(d)

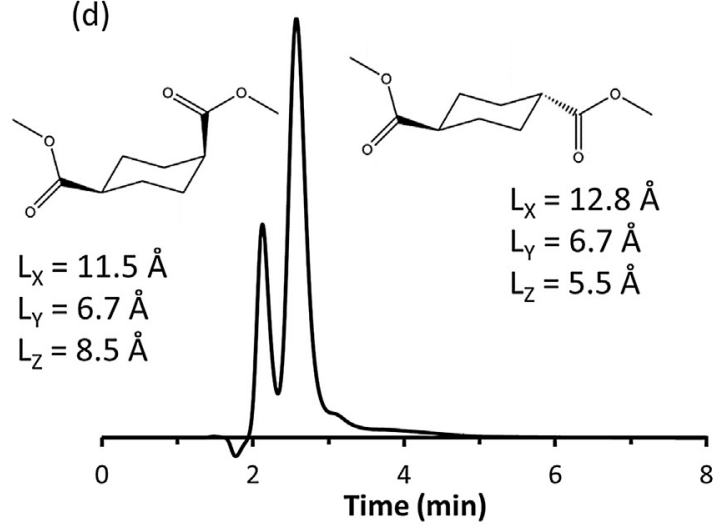

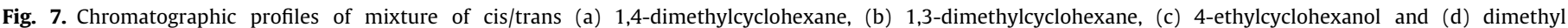

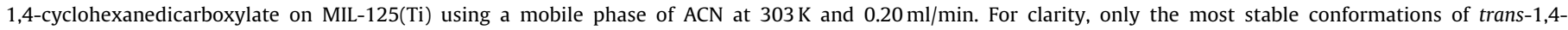

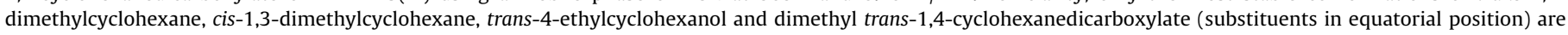
shown, together with their molecular dimensions.

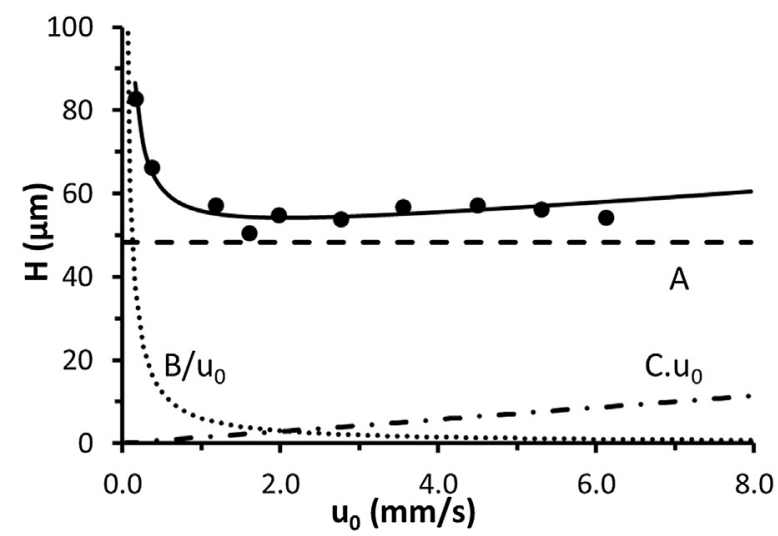

Fig. 8. van Deemter plot of benzene on MIL-125(Ti) using a mobile phase of ACN at $303 \mathrm{~K}$, showing experimental $\mathrm{H}$ values $(\bullet)$ and a fitting of the van Deemter equation through these points (solid line) with the contributions of the A- (dashed line), B(dotted line) and C-term (dash dotted line).

plot to determine the optimal mobile phase velocity [32]. So far, only MOF based stationary phases in GC [46-50] and monolith-MOF columns in HPLC [51-53] were characterized using a van Deemter plot. As shown in Fig. 8, the van Deemter plot for benzene shows a minimum plate height $\mathrm{H}$ of about $50 \mu \mathrm{m}$ at a flow rate between 0.20 and $0.25 \mathrm{ml} / \mathrm{min}$ (Fig. 8). For commercial HPLC columns, a minimum plate height of 2 times the particle diameter is indicative for very good packing and particle monodispersity [54]. Regarding the less regular particle shape and size of MIL-125(Ti) (Fig. 1b) and the non-optimized slurry packing method, this minimum plate height
Table 2

Fitting data of the van Deemter coefficients for benzene on MIL-125(Ti), from which minimal plate height $\mathrm{H}_{\min }$, optimal linear velocity $\mathrm{u}_{0, \mathrm{opt}}$ and optimal flow rate $\mathrm{F}_{\mathrm{opt}}$ is calculated.

\begin{tabular}{cc}
\hline $\mathrm{A}(\mu \mathrm{m})$ & 48.3 \\
$\mathrm{~B}\left(\mathrm{~mm}^{2} / \mathrm{s}\right)$ & $6.08 \times 10^{-3}$ \\
$\mathrm{C}(\mathrm{s})$ & $1.43 \times 10^{-3}$ \\
$\mathrm{H}_{\min }(\mu \mathrm{m})$ & 54.2 \\
$\mathrm{u}_{0, \mathrm{opt}}(\mathrm{mm} / \mathrm{s})$ & 1.97 \\
$\mathrm{~F}_{\mathrm{opt}}(\mathrm{ml} / \mathrm{min})$ & 0.25 \\
\hline
\end{tabular}

is acceptable. Nevertheless, in comparison with commercial HPLC columns with similarly sized spherical particles (i.e. circa $3 \mu \mathrm{m}$ ), the minimum plate height of the MIL-125(Ti) column is about one order of magnitude larger. If this minimum plate height is converted to number of plates per m, an efficiency of about 20,000 plates $/ \mathrm{m}$ is reached for benzene, competing with the performance of MOF-silica core-shell particles [14-16].

The fitted van Deemter coefficients are shown in Table 2. The high contribution of the A-term, which is an indication of the heterogeneity of a packed bed, can be ascribed to the polydispersity of the MOF crystals (certainly in comparison with commercial silica particles) and their not-spherical shape. The low contribution of the C-term (in the case of benzene, see Table 2) allows to perform certain separations at elevated flow rates. Thus, separation time can be reduced without losing too much efficiency.

\section{Conclusions}

The adsorption and separation properties of the MIL-125(Ti) in liquid chromatography have been studied for different kinds of 
adsorbates. The structure contains both small tetrahedral cages and large octahedral cages, playing a fundamental role in the shape selective separation of molecules with similar size. It was found that molecular dimensions play a crucial role in the adsorption behavior: elongated molecules with a small width are preferred over shorter but more bulky molecules, as noted for aromatic positional isomers and PAHs, as a result of a better fit and interaction with the small tetrahedral cages. Besides the steric effects, the presence of hydroxyl groups $\left(\mu_{2}-\mathrm{OH}\right)$ in the MIL-125(Ti) structure enhances the interaction between specific analytes and the framework, resulting in an interplay between shape selective effects (in the smallest cages) and enthalpic effects. This allows for very specific separations, especially of mixtures of small molecules which are difficult to separate using classical mesoporous solids. For example, stereoisomer selectivity for cis/trans functionalized cyclohexane isomers was demonstrated, with a clear preference for the trans configuration in most cases.

Furthermore, the van Deemter curve revealed an acceptable minimum plate height $( \pm 50 \mu \mathrm{m})$ with a significant contribution of the A-term, reflecting the bed heterogeneity of the self-packed column. The MIL-125(Ti) structure could be a potential separation medium as stationary phase in liquid chromatographic applications, offering perspectives for stereoselective separations.

\section{Acknowledgements}

The authors are grateful to the Agency of Innovation by Science and Technology Flanders (IWT) for support in SBO project MOFShape. J.D. and D.D.V. are also grateful to FWO for project support (G.0256.14N).

\section{Appendix A. Supplementary data}

Supplementary data associated with this article can be found, in the online version, at http://dx.doi.org/10.1016/j.chroma.2016.09. 057.

\section{References}

[1] Y. Yu, Y. Ren, W. Shen, H. Deng, Z. Gao, Applications of metal-organic frameworks as stationary phases in chromatography, TrAC Trends Anal. Chem. 50 (2013) 33-41.

[2] K. Yusuf, A. Aqel, Z. Alothman, Metal-organic frameworks in chromatography, J. Chromatogr. A 1348 (2014) 1-16.

[3] R. Ahmad, A.G. Wong-Foy, A.J. Matzger, Microporous coordination polymers as selective sorbents for liquid chromatography, Langmuir 25 (2009) 11977-11979.

[4] L. Alaerts, M. Maes, M.A. van der Veen, P.A. Jacobs, D.E. De Vos, Metal-organic frameworks as high-potential adsorbents for liquid-phase separations of olefins, alkylnaphthalenes and dichlorobenzenes, Phys. Chem. Chem. Phys. 11 (2009) 2903-2911.

[5] C.-X. Yang, S.-S. Liu, H.-F. Wang, S.-W. Wang, X.-P. Yan, High-performance liquid chromatographic separation of position isomers using metal-organic framework MIL-53(Al) as the stationary phase, Analyst 137 (2012) 133-139.

[6] W. De Malsche, S. Van der Perre, S. Silverans, M. Maes, D.E. De Vos, F. Lynen, J.F. Denayer, Unusual pressure-temperature dependency in the capillary liquid chromatographic separation of C8 alkylaromatics on the MIL-53(Al) metal-organic framework, Micropor. Mesopor. Mater. 162 (2012) 1-5.

[7] R. El Osta, A. Carlin-Sinclair, N. Guillou, R.I. Walton, F. Vermoortele, M. Maes, D. de Vos, F. Millange, Liquid-phase adsorption and separation of xylene isomers by the flexible porous metal-organic framework MIL-53(Fe), Chem. Mater. 24 (2012) 2781-2791.

[8] Y.-Y. Fu, C.-X. Yang, X.-P. Yan, Control of the coordination status of the open metal sites in metal-organic frameworks for high performance separation of polar compounds, Langmuir 28 (2012) 6794-6802.

[9] Y.-Y. Fu, C.-X. Yang, X.-P. Yan, Metal-organic framework MIL-100(Fe) as the stationary phase for both normal-phase and reverse-phase high performance liquid chromatography, J. Chromatogr. A 1274 (2013) 137-144.

[10] C.-X. Yang, X.-P. Yan, Metal-organic framework MIL-101(Cr) for high-performance liquid chromatographic separation of substituted aromatics, Anal. Chem. 83 (2011) 7144-7150.

[11] C.-X. Yang, Y.-J. Chen, H.-F. Wang, X.-P. Yan, High-performance separation of fullerenes on metal-organic framework MIL-101(Cr), Chem. Eur. J. 17 (2011) 11734-11737.
[12] F. Vermoortele, M. Maes, P.Z. Moghadam, M.J. Lennox, F. Ragon, M. Boulhout, S. Biswas, K.G.M. Laurier, I. Beurroies, R. Denoyel, M. Roeffaers, N. Stock, T. Düren, C. Serre, D.E. De Vos, p-Xylene-selective metal-organic frameworks: a case of topology-directed selectivity, J. Am. Chem. Soc. 133 (2011) 18526-18529.

[13] M.A. Moreira, J.C. Santos, A.F. Ferreira, J.M. Loureiro, F. Ragon, P. Horcajada P.G. Yot, C. Serre, A.E. Rodrigues, Toward understanding the influence of ethylbenzene in p-Xylene selectivity of the porous titanium amino terephthalate MIL-125(Ti): adsorption equilibrium and separation of xylene isomers, Langmuir 28 (2012) 3494-3502.

[14] S. Van der Perre, T. Duerinck, P. Valvekens, D.E. De Vos, G.V. Baron, J.F.M Denayer, Chromatographic separation through confinement in nanocages, Micropor. Mesopor. Mater. 189 (2014) 216-221.

[15] R. Ameloot, A. Liekens, L. Alaerts, M. Maes, A. Galarneau, B. Coq, G. Desmet, B.F. Sels, J.F.M. Denayer, D.E. De Vos, Silica-MOF composites as a stationary phase in liquid chromatography, Eur. J. Inorg. Chem. 2010 (2010) 3735-3738.

[16] A. Ahmed, M. Forster, R. Clowes, D. Bradshaw, P. Myers, H. Zhang, Silica SOS@HKUST-1 composite microspheres as easily packed stationary phases for fast separation, J. Mater. Chem. A 1 (2013) 3276-3286.

[17] Y.-Y. Fu, C.-X. Yang, X.-P. Yan, Fabrication of ZIF-8@SiO 2 Core-Shell microspheres as the stationary phase for high-performance liquid chromatography, Chem. Eur. J. 19 (2013) 13484-13491.

[18] A. Ahmed, M. Forster, J. Jin, P. Myers, H. Zhang, Tuning morphology of nanostructured ZIF-8 on silica microspheres and applications in liquic chromatography and dye degradation, ACS Appl. Mater. Interfaces 7 (2015) 18054-18063.

[19] Z. Yan, J. Zheng, J. Chen, P. Tong, M. Lu, Z. Lin, L. Zhang, Preparation and evaluation of silica-UiO-66 composite as liquid chromatographic stationary phase for fast and efficient separation, J. Chromatogr. A 1366 (2014) 45-53.

[20] X. Zhang, Q. Han, M. Ding, One-pot synthesis of UiO-66@ $\mathrm{SiO}_{2}$ shell-core microspheres as stationary phase for high performance liquid chromatography, RSC Adv. 5 (2015) 1043-1050.

[21] K. Tanaka, T. Muraoka, D. Hirayama, A. Ohnish, Highly efficient chromatographic resolution of sulfoxides using a new homochiral MOF-silica composite, Chem. Commun. 48 (2012) 8577-8579.

[22] M. Dan-Hardi, C. Serre, T. Frot, L. Rozes, G. Maurin, C. Sanchez, G. Férey, A new photoactive crystalline highly porous titanium(IV) dicarboxylate, J. Am. Chem. Soc. 131 (2009) 10857-10859.

[23] S. Hu, M. Liu, K. Li, Y. Zuo, A. Zhang, C. Song, G. Zhang, X. Guo, Solvothermal synthesis of $\mathrm{NH}_{2}-\mathrm{MIL}-125$ (Ti) from circular plate to octahedron, CrystEngComm 16 (2014) 9645-9650.

[24] N.A. Ramsahye, P. Trens, C. Shepherd, P. Gonzalez, T.K. Trung, F. Ragon, C. Serre, The effect of pore shape on hydrocarbon selectivity on UiO-66(Zr) HKUST-1 and MIL-125(Ti) metal organic frameworks: insights from molecular simulations and chromatography, Micropor. Mesopor. Mater. 189 (2014) 222-231.

[25] T. Duerinck, R. Bueno-Perez, F. Vermoortele, D.E. De Vos, S. Calero, G.V. Baron, J.F.M. Denayer, Understanding hydrocarbon adsorption in the UiO-66 metal-organic framework: separation of (Un)saturated linear, branched, cyclic adsorbates, including stereoisomers, J. Phys. Chem. C 117 (2013) $12567-12578$

[26] T. Duerinck, J.M. Denayer, Unusual chain length dependent adsorption of linear and branched alkanes on UiO-66. Adsorption 20 (2014) 251-259.

[27] S.-N. Kim, J. Kim, H.-Y. Kim, H.-Y. Cho, W.-S. Ahn, Adsorption/catalytic properties of MIL-125 and NH2-MIL-125, Catal. Today 204 (2013) 85-93.

[28] Y. Zhang, Y. Chen, Y. Zhang, H. Cong, B. Fu, S. Wen, S. Ruan, A novel humidity sensor based on $\mathrm{NH}_{2}-\mathrm{MIL}-125(\mathrm{Ti})$ metal organic framework with high responsiveness, J. Nanopart. Res. 15 (2013) 1-6.

[29] Y. Zhang, B. Fu, K. Liu, Y. Zhang, X. Li, S. Wen, Y. Chen, S. Ruan, Humidity sensing properties of $\mathrm{FeCl}_{3}-\mathrm{NH}_{2}-\mathrm{MIL}-125$ (Ti) composites, Sens. Actuators B: Chem. 201 (2014) 281-285.

[30] A. Schaate, P. Roy, A. Godt, J. Lippke, F. Waltz, M. Wiebcke, P. Behrens, Modulated synthesis of $\mathrm{Zr}$-based metal-organic frameworks: from nano to single crystals, Chem. Eur. J. 17 (2011) 6643-6651.

[31] T. Frot, S. Cochet, G. Laurent, C. Sassoye, M. Popall, C. Sanchez, L. Rozes, $\mathrm{Ti}_{8} \mathrm{O}_{8}(\mathrm{OOCR})_{16}$, a new family of titanium-Oxo clusters: potential NBUs for reticular chemistry, Eur. J. Inorg. Chem. 2010 (2010) 5650-5659.

[32] J.J. van Deemter, F.J. Zuiderweg, A. Klinkenberg, Longitudinal diffusion and resistance to mass transfer as causes of nonideality in chromatography, Chem. Eng. Sci. 5 (1956) 271-289.

[33] E. Grushka, N. Grinberg, Advances in Chromatography, CRC Press, 2012.

[34] S. Vaesen, V. Guillerm, Q. Yang, A.D. Wiersum, B. Marszalek, B. Gil, A. Vimont, M. Daturi, T. Devic, P.L. Llewellyn, C. Serre, G. Maurin, G. De Weireld, A robust amino-functionalized titanium(iv) based MOF for improved separation of acid gases, Chem. Commun. 49 (2013) 10082-10084

[35] C. Serre, S. Bourrelly, A. Vimont, N.A. Ramsahye, G. Maurin, P.L. Llewellyn, M Daturi, Y. Filinchuk, O. Leynaud, P. Barnes, G. Férey, An explanation for the very large breathing effect of a metal-organic framework during $\mathrm{CO}_{2}$ adsorption, Adv. Mater. 19 (2007) 2246-2251.

[36] S. Couck, J.F.M. Denayer, G.V. Baron, T. Remy, J. Gascon, F. Kapteijn, An amine-functionalized MIL-53 metal-organic framework with large separation power for $\mathrm{CO}_{2}$ and $\mathrm{CH}_{4}$, J. Am. Chem. Soc. 131 (2009) 6326-6327.

[37] S. Bourrelly, B. Moulin, A. Rivera, G. Maurin, S. Devautour-Vino, C. Serre, T. Devic, P. Horcajada, A. Vimont, G. Clet, M. Daturi, J.C. Lavalley, S. Loera-Serna, R. Denoyel, P.L. Llewellyn, G. Ferey, Explanation of the adsorption of polar 
vapors in the highly flexible metal organic framework MIL-53(Cr), J. Am. Chem. Soc. 132 (2010) 9488-9498.

[38] E. Heftmann, Chromatography: fundamentals and applications of chromatography and related differential migration methods - Part A: fundamentals and techniques, Elsevier Sci. (2004).

[39] C. Horváth, High-performance Liquid Chromatography: Advances and Perspectives, Elsevier, 2013

[40] T. Duerinck, S. Couck, F. Vermoortele, D.E. De Vos, G.V. Baron, J.F.M. Denayer, Pulse gas chromatographic study of adsorption of substituted aromatics and heterocyclic molecules on MIL-47 at zero coverage, Langmuir 28 (2012) $13883-13891$.

[41] L.C. Gilday, S.W. Robinson, T.A. Barendt, M.J. Langton, B.R. Mullaney, P.D. Beer, Halogen bonding in supramolecular chemistry, Chem. Rev. 115 (2015) $7118-7195$.

[42] X. Shu, J. Fan, J. Li, X. Wang, W. Chen, X. Jia, C. Li, Complexation of neutral 1,4-dihalobutanes with simple pillar[5] arenes that is dominated by dispersion forces, Org. Biomol. Chem. 10 (2012) 3393-3397.

[43] S. Gobolos, Z. Banka, Z. Toth, J. Szammer, J.L. Margitfalvi, Highly Selective Preparation of trans-4-Aminocyclohexane Carboxylic Acid from cis-Isomer over Raney ${ }^{\circledR}$ Nickel Catalyst, Chemical industries-new york then boca raton-marcel dekker then CRC Press, 115, 2007, 45.

[44] N.K. Pandit, Introduction to the Pharmaceutical Sciences, Lippincott Williams \& Wilkins, 2007.

[45] W.O. Foye, T.L. Lemke, D.A. Williams, Foye's Principles of Medicinal Chemistry, Lippincott Williams \& Wilkins, 2008.

[46] A.S. Münch, J. Seidel, A. Obst, E. Weber, F.O.R.L. Mertens, High-separation performance of chromatographic capillaries coated with MOF- 5 by the controlled SBU approach, Chem. Eur. J. 17 (2011) 10958-10964.
[47] S.-M. Xie, Z.-J. Zhang, Z.-Y. Wang, L.-M. Yuan, Chiral metal-Organic frameworks for high-resolution gas chromatographic separations, J. Am. Chem. Soc. 133 (2011) 11892-11895.

[48] L. Fan, X.-P. Yan, Evaluation of isostructural metal-organic frameworks coated capillary columns for the gas chromatographic separation of alkane isomers, Talanta 99 (2012) 944-950.

[49] T. Böhle, F. Mertens, Two isoreticular pillared-layer frameworks as stationary phases for gas chromatographic applications - unusual peak broadening in size exclusion chromatography, determination of thermodynamic and kinetic data, Micropor. Mesopor. Mat. 216 (2015) 82-91.

[50] X. Xue, M. Zhang, S. Xie, L. Yuan, Homochiral metal-organic framework $\left[\mathrm{Zn}_{2} \text { (d-Cam }\right)_{2}\left(4,4^{\prime}\right.$-bpy) $] \mathrm{n}$ for high-resolution gas chromatographic separations, Acta Chromatogr. 27 (2015) 15-26.

[51] H.-Y. Huang, C.-L. Lin, C.-Y. Wu, Y.-J. Cheng, C.-H. Lin, Metal organic framework-organic polymer monolith stationary phases for capillary electrochromatography and nano-liquid chromatography, Anal. Chim. Acta 779 (2013) 96-103.

[52] S. Yang, F. Ye, Q. Lv, C. Zhang, S. Shen, S. Zhao, Incorporation of metal-organic framework HKUST-1 into porous polymer monolithic capillary columns to enhance the chromatographic separation of small molecules, J. Chromatogr. A 1360 (2014) 143-149.

[53] S. Yang, F. Ye, C. Zhang, S. Shen, S. Zhao, In situ synthesis of metal-organic frameworks in a porous polymer monolith as the stationary phase for capillary liquid chromatography, Analyst 140 (2015) 2755-2761.

[54] K. Broeckhoven, D. Cabooter, G. Desmet, Kinetic performance comparison of fully and superficially porous particles with sizes ranging between $2.7 \mu \mathrm{m}$ and $5 \mu \mathrm{m}$ : intrinsic evaluation and application to a pharmaceutical test compound, J. Pharm. Anal. 3 (2013) 313-323. 